\title{
Modelling and Development of a Pseudo-Hydraulic Power Steering Model for use in Real-Time Applications
}

\author{
Theodor Ensbury ${ }^{1}$ Peter Harman ${ }^{2}$ Mike Dempsey ${ }^{1}$ \\ ${ }^{1}$ Claytex Services Ltd. Edmund House, Rugby Road, Leamington Spa, CV32 6EL, UK \\ \{theodor.ensbury, mike.dempsey\} dclaytex.com \\ ${ }^{2}$ CAE Tech Limited, Leamington Spa, UK, peter. harman@cae. tech
}

\begin{abstract}
Driver-in-the-loop (DiL) simulation is playing an increasing role in automotive OEM development processes. Vehicle models used in these activities therefore need to be as accurate, and realistic, as possible. This paper will present the modelling and development of a pseudo-hydraulic power steering model, designed for usage in DiL applications. Specific focus during development has been towards the quantification and analysis of the torque feedback from the steering model to the simulator rig steering wheel, to produce as realistic a steering 'feel' as possible. Metrics derived from physical testing of vehicle steering systems have been deployed to analyze the torque feedback of the steering system. Subsequent assessment of the steering model and specific parameterization has been used to inform the model parameters utilized. Results quantifying the performance of the steering model during full vehicle testing using the Claytex VeSyMA platform are presented.
\end{abstract}

Keywords: Driver-in-the-loop, DiL, steering feel, hydraulic power steering

\section{Introduction}

As real-time Driver-in-the-loop (DiL) vehicle simulation has increased in deployment across the automotive industry, the need for accurate models correctly depicting qualitative aspects of vehicle behavior, has increased. Vehicle steering, as one qualitive aspect of vehicle experience, should be as close to real life as possible for driver immersion (Ansible Motion, 2015). To understand why, the role steering torque feedback plays in the driver/vehicle interaction must be considered.

Despite the recent trend of OEMS moving to fully electric systems, some, such as Nissan, state preference for using hydraulic assistance systems based on a perception of superior feel. This has led to the development of hybrid electro-hydraulic systems, with an electric pump assisting the steering through hydraulic fluid (Nissan, 2018). Thus, it is important to model the dynamics of the hydraulic assistance system to capture the steering feel. Therefore, the object of this paper is not to present a model of an electro-hydraulic steering system, but rather to present a pseudo-physical steering model accounting for the dynamics of hydraulic assistance, such as the decay rate of force when the torque applied drops off suddenly, without the numerical complexity of a fully physical system model.

\subsection{Impact of steering feel in DiL applications}

When driving within a DiL environment, the driver senses what the vehicle model is doing through several senses; one of these is haptic (touch). This concerns the human/physical interface of which the steering forms a part (Ansible Motion, 2015). Essentially, the steering in a DiL simulator is a haptic feedback device. As a primary feedback on vehicle behavior, correct steering feel is therefore important to enable the driver to control the vehicle in as realistic method as possible. Dynamic limit control of the vehicle by the driver is therefore impacted by the accuracy of the steering feel. This applies to DiL simulation in both handling studies and driver/vehicle control system interaction, such as electronic stability program (ESP) development. For ESPs to be correctly developed, the driver must thus react as realistically as possible given the confines of a simulation environment (Ansible Motion, 2015). Accuracy of the steering model must therefore be retained in both normal driving condition, characterized by on-center driving (Pfeffer et al. 2008), as well as offcenter scenarios.

\subsection{Real-time modelling constraints}

As the target application of the steering model will be in DiL simulation scenarios, the steering model utilized must therefore be fast enough to run in real-time. By nature, hydraulic power steering torque feedback displays a non-linear hysteretic characteristic. Previous implementations of steering models in detailed real-time scenarios have required the use of external models devoted towards modelling the steering (Andreasson, et al. 2016). Whilst this is one solution to capturing the correct characteristics in a computationally efficient manor, a steering model integral to the vehicle model is a preferable solution with regards ease of use by the model user.

The vehicle model utilized to test, develop and parameterize the steering model must also be of required quality and run fast enough for smooth real-time running. As the steering feel and vehicle response to 


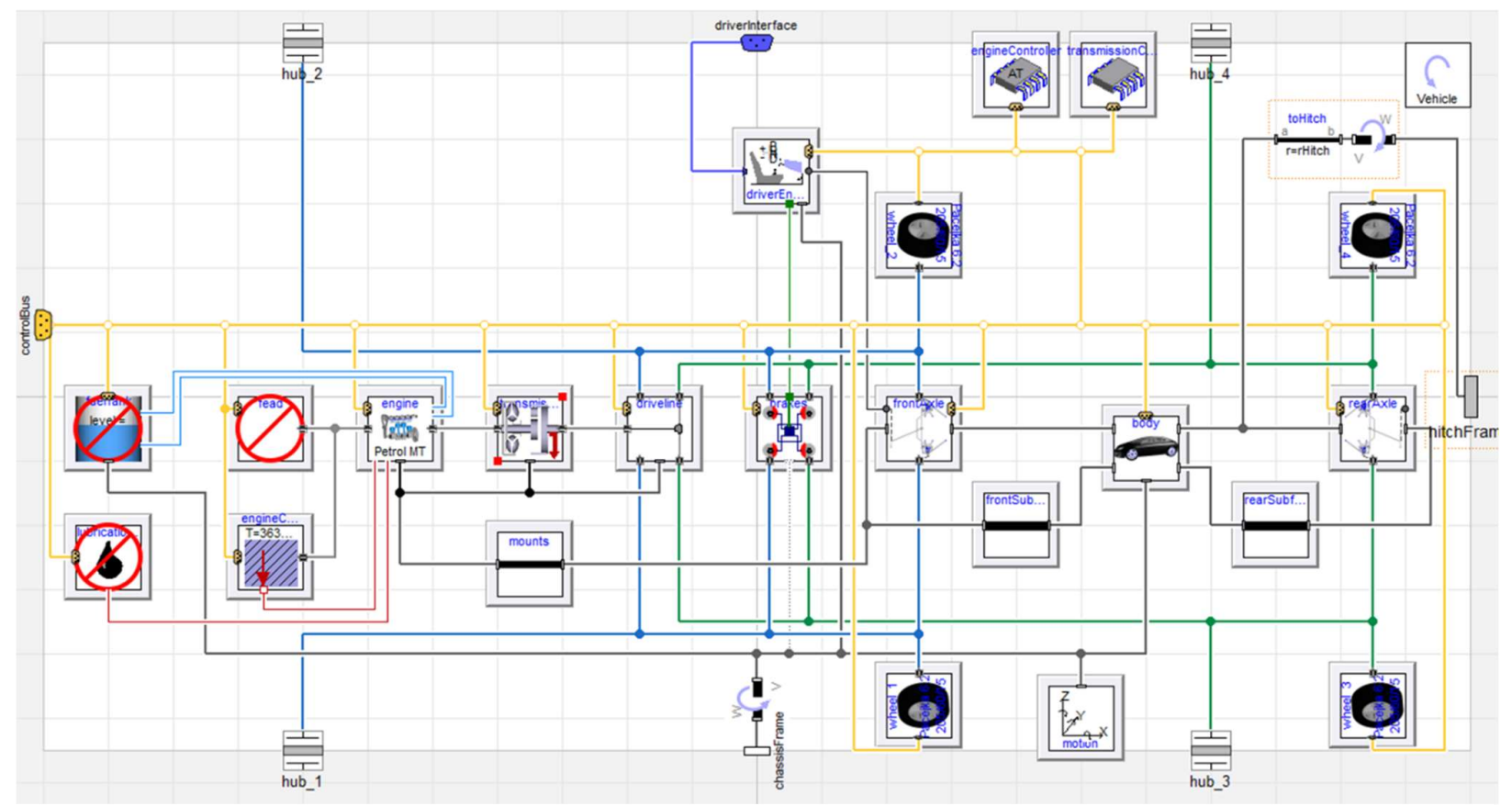

Figure 1. The vehicle model built to test and develop the Pseudo-Hydraulic power steering model. Built from a template from the VeSyMA library, this model features subsystems and components from the VeSyMA, VeSyMA - Suspensions libraries. Note, steering wheel connection is handled acausaly through the driver interface.

steering input are interlinked (Pfeffer et al. 2008), it is paramount the vehicle and tire model are of sufficient fidelity with respect to the analysis for the study to be valid. Due to the consideration in this paper of the transient response of the steering/vehicle, a transient, non-linear vehicle and tire model is required. The object-oriented nature of Modelica enables the vehicle model to be tailored to the application, therefore simplified systems for vehicle aspects such as the powertrain can be deployed.

\section{Modelling}

The steering model described in this paper, as well as the vehicle model and vehicle test used in the development and analysis of the steering model form part of the Claytex VeSyMA suite of libraries.

\subsection{Overview of Claytex VeSyMA suite}

Developed for use with the Dymola simulation environment, the Vehicle Systems Modelling and Analysis (VeSyMA) suite is a complete vehicle simulation solution built from Modelica. Utilizing the object-oriented nature of the Modelica language, the principle of the VeSyMA suite is several separate subject specific libraries all of which are compatible with one another, as they share a common parent, the VeSyMA library, which defines vehicle level templates. As the open source Vehicle Interfaces library provides the basis of the templates in the VeSyMA library, third party models can be capable of being compatible with VeSyMA vehicle models.
On its own, the VeSyMA library is capable of straight line vehicle analysis; each subsequent subject specific library adds in fidelity and complexity to the overall model. Examples of subject libraries include the VeSyMA - Suspensions library, which defines 3D multibody suspension systems, road models and highfidelity tire models, or the VeSyMA - Engines library which defines various high-fidelity powertrain components. A schematic overview of the VeSyMA suite is presented in Figure 2.

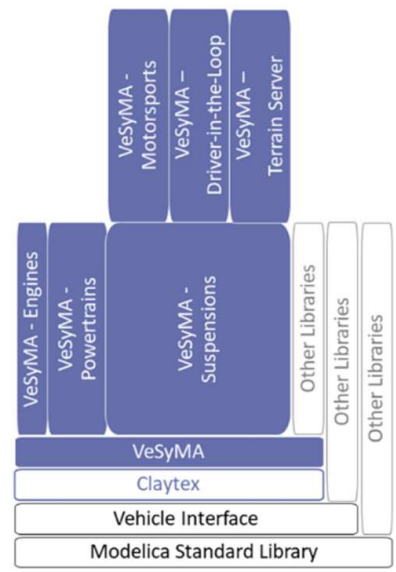

Figure 2. Schematic overview of the VeSyMA suite of libraries from Clayex. Note, the VeSyMA - Suspensions, VeSyMA - Driver-in-the-Loop and VeSyMA libraries were utilized in the study this paper describes. 


\subsection{Vehicle model}

A complete multibody vehicle model utilized to test and develop the pseudo-hydraulic power steering model, free to move within the simulation environment in all 6 degrees (lateral, longitudinal, heave; roll, pitch and yaw) is presented as Figure 1. All elements within the vehicle model were taken from the VeSyMA or VeSyMA Suspensions libraries. The vehicle platform employed was loosely based upon a passenger car, specifically the executive/E class. Double wishbone suspension was used for both the front and rear axles of the vehicle, with drive torque sent to the rear wheels as part of a standard rear-wheel drive (RWD) layout. No electronic stability programs, active yaw control or other electronic driver aids were used in the vehicle.

The vehicle model is required to be of sufficient fidelity, whilst simultaneously being as computationally efficient as possible to be capable of real-time DiL running. Compromises were therefore made regarding the fidelity of non-essential components. Specifically, idealized powertrain components were used, including:

- Mapped engine model

- Idealized paddle shift transmission model

- No lubrication or FEAD systems

- 1D rotational driveline model

Compliances within the suspension mounting points were also omitted and a simplified aerodynamic model detailing the lift, side and drag forces used. A fourwheel disc brake model with a controlled elasto-plastic friction model (Dankowicz, 1999) was also used.

Priority was given to the fidelity of the suspensions and tire models employed within the vehicle model. Full multibody suspension linkages were used, featuring Claytex aggregate joints in place of standard spherical joints; aggregate joints are computationally more efficient than standard multibody joints, developed specifically for real-time applications. The block diagram of the suspension linkage model used is presented as Figure 3.

Each link within the suspension model included specific mass and inertial properties, but linkage mounting compliance or suspensions member flex was omitted. Translation force elements were deployed as the ride springs and dampers, connected directly to the lower control arm. Full multibody anti-roll bars were also utilized on both the front and rear axles.

A fully combined lateral and longitudinal slip tire model was also used, therefore the lateral and longitudinal forces produced by the tires are non-linear. This comprised of a Modelica implementation of the Pacejka Magic Formula (corresponding to MF6.2). Included were asymmetric tire behavior due conicity and ply-steer; a kelvin spring damper modelled the vertical dynamics of the tire (Pacejka, 2012).

An explicit Runga-Kutta time integration method with a fixed time step of $1 \mathrm{~m} / \mathrm{s}$ was utilized for both the

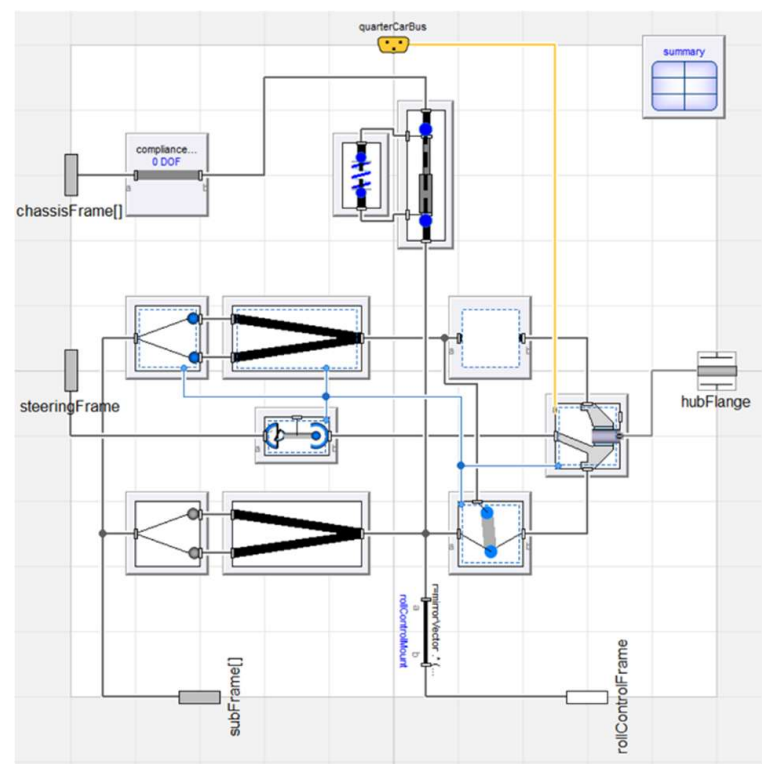

Figure 3. Double wishbone Quarter Car linkage model deployed once per wheel in the frontAxle model. Note the use of the aggreagted joints in place of spherical joints.

steering model study and real-time running. Approximately 100 time-states were present in the translated vehicle model.

\subsection{Steering model concept}

Pfeffer et al. (2008) presented a steering model designed to capture the correct feel of the vehicle steering during on-center driving, due to the dominance of this range in the experience of daily road drivers. Lateral dynamics up to $4 \mathrm{~ms}^{-1}$ were deemed to be of interest in their study. Validation of the model presented was achieved against vehicle measurements from testing a BMW E46 with a hydraulic power steering system; therefore, this steering model presents a robust basis for steering model development. Furthermore, computational concerns were considered during modeling by Pfeffer et al. (2008) further increasing the suitability of the model to the application described in this paper.

The basic premise of the Pfeffer et al. (2008) model is to split the steering system into two sections; mechanical and hydraulic. Work on the hydraulic element of the steering model presented in this paper is presented in section 2.4. Comprising the mechanical elements of the steering model are the column and the rack with pinion. Thus, the mechanical system has 2 degrees of freedom, one rotational (column) and one translational (rack). Inertias for the two mechanical elements are considered separate, with the compliance of the steering system modelling as part of the column. Figure 4 presents the holistic steering model developed for this paper. Of note is the lack of upper column and steering wheel inertia shown in Figure 4; this is present and modelled separately (but rigidly connected) to the steering model shown during offline simulations, but it 
is replaced with the physical column and wheel in simulator rigs. Inertia below the column compliance is modelled in this steering model. Power assistance is applied to the steering system at the rack in the Pfeffer et al. (2008) model.

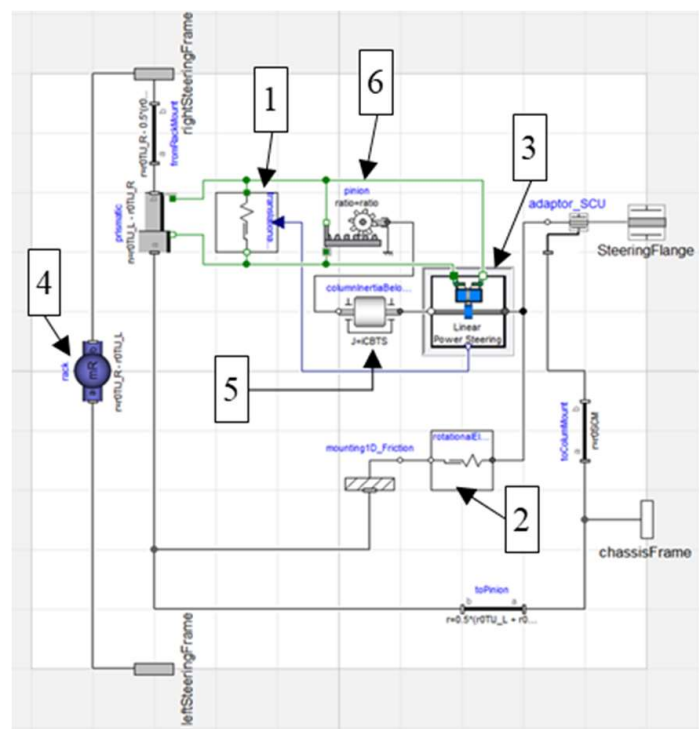

Figure 4. Steering model developed for this paper, deployed as part of the frontAxle model. Section 2.4 details the Linear Power Steering block, featuring the pseudohydraulic model. Notes: 1 is the rack friction model, 2 is the column friction model, 3 is the pseudo-hydraulic power assistance model, 4 is the rack mass, 5 is the column inertia below the compliance, 6 is the pinion/rack model. The upper column inertia is modelled externally to this model, rigidly connected to the steeringFlange.

As during physical operation both the column and the rack move within their respective housing, they cannot be considered ideal in motion. Therefore, friction has been included by separate models for both the column and the rack. Friction losses between the pinion gear and the rack are considered as part of the rack friction model. Pfeffer et al. (2008) utilized Exponential-SpringFriction-Elements (ESF-Element) in their work to model the friction effects on the column and rack. A key aspect of the ESF-Element friction models is the dynamic state behavior. This is to capture the hysteretic characteristic required, resulting from the differing sliding and pre-sliding aspects of dynamic and static friction present within a steering system.

However, a changing state behavior renders them unsuitable for real-time simulation, due to the computational penalty associated with state events during time integration. A single-state friction model must therefore be utilized to avoid introducing events. Dupont et al. (2002). presented an 'elasto-plastic' single state friction model suitable for use in real-time applications. Termed 'elasto-plastic', the model developed by Dupont et al. (2002) differs from standard single state friction models (such as Dahl or LuGre). In this model, the presiding behavior is modeled in an elastic method initially before transitioning into plastic behavior, hence the term 'elasto-plastic' being coined. As the elastic component is reversible (whereas the plastic is irreversible), it is argued the total drift (deemed "spurious") of the elasto-plastic friction during presliding is greatly reduced compared to other single state models, which rely solely on plastic behavior during pre-sliding. It is stated that the elasto-plastic model is therefore valid in periodic conditions dominated by presliding; conditions a vehicle steering system experiences, as proved by the hysteretic behavior displayed during on-center driving.

\subsection{Pseudo-hydraulic power assistance model}

The model relating the steering column dynamics to the assistance force is termed 'pseudo-hydraulic', as it aims to capture the key dynamics of a hydraulic power steering but without physical modelling of the internal elements of that system.

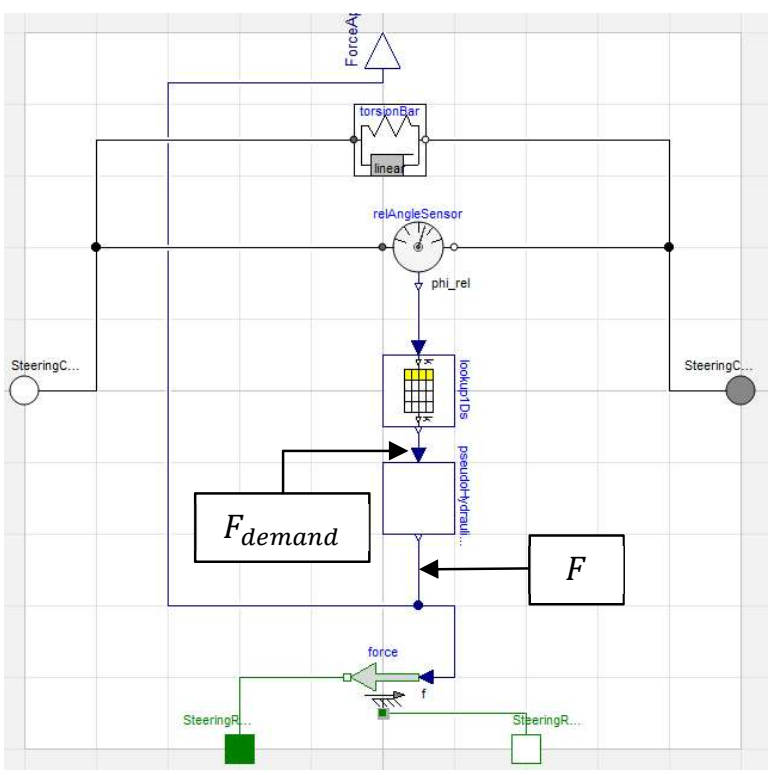

Figure 5. Inside the Linear Power Steering block. The rotational flanges of the steering-column are connected by the torsion-bar. The relative angle is used to calculate the assistance force demand, with the assistance force generated by the pseudo-hydraulic block.

The assistance force from a hydraulic power steering system is applied by a linear actuator with two chambers. The force is calculated from the pressure difference in the chambers. This actuator is connected to a spool valve, which can be either linear or rotary, that connects the chambers to either a high supply pressure from the pump or a low return pressure to tank. The steering column contains a torsion-bar giving compliance between handwheel angle and the rack. It is the relative angle across this torsion-bar that opens and 
closes the spool. In Figure 5, we have connected a relative angle sensor to a lookup table, which contains the steady-state assistance force demand for the torsionbar twist. Assistance force is then produced by the pseudo-hydraulic block relative to the assistance demand.

If we neglect non-linearities due to fluid viscosity and compressibility effects, the rate-of-change of the fluid pressure is proportional to the square-root of the pressure-drop across the spool. The effect of the profile of the spool is incorporated in the steady-state assistance force table, and hence our model only needs to calculate an actual assistance force based on a demanded assistance force. A typical power-steering valve comprises a rotary spool, with a bevel profile that smooths the transition of opening area. Identification and modelling of spool geometry is described in detail by Rösth (2007).

Critical to the steering feel is the decay in assistance force. This differs from the rate of increase due to the internal flow areas in the hydraulic system. We therefore define 2 parameters for a minimal model of hydraulic behavior.

- rate $[1 / \mathrm{s}]$ : The gain from the force demand to rateof-change of force from one side of the actuator

- emptyingFactor [1]: The factor of emptying rate vs filling rate, 1 means equal, 2 means twice as fast to empty (and force to decay)

The variables involved in the model are divided into Left and Right, as they are equivalent to the chambers in the actuator.

- $\mathrm{F}_{\text {demand }}[\mathrm{N}]$ : The steady-state assistance force

- $\mathrm{F}_{\text {demandL }}[\mathrm{N}]$ : Demand on the left

- $\mathrm{F}_{\text {demandR }}[\mathrm{N}]$ : Demand on the right

- $\mathrm{F}_{\mathrm{L}}[\mathrm{N}]$ : The force on the left

- $\mathrm{F}_{\mathrm{R}}[\mathrm{N}]$ : The force on the right

- $\mathrm{F}[\mathrm{N}]$ : The assistance force applied to the steering rack

The equations are as follows:

$$
\begin{gathered}
F_{\text {demand } L}=\max \left(F_{\text {demand }}, F_{L}\right)-F_{L} \\
F_{\text {demand } R}=\max \left(-F_{\text {demand }}, F_{R}\right)-F_{R} \\
\left.\frac{d F_{L}}{d t}=\text { rate } \sqrt{F_{\text {demandL }}(1+\text { emptyingFactor }}{ }^{2}\right) \\
- \text { emptyingFactor } \sqrt{F_{L}} \\
\frac{d F_{R}}{d t}=\text { rate } \sqrt{F_{\text {demand } R}\left(1+\text { emptyingFactor }{ }^{2}\right)} \\
- \text { emptyingFactor } \sqrt{F_{R}} \\
F=F_{L}-F_{R}
\end{gathered}
$$

Due to square-roots having an infinite derivative at zero, the implementation uses an approximation with a finite derivative to help the solver performance. There is therefore a $3^{\text {rd }}$ parameter in the model, delta, which defines the closeness to the true square-root function.

\section{Steering feel quantification}

Objective analysis of steering hysteresis loop is a challenging task, therefore attempting to quantify steering feel from looking at links between subjective ratings and objective parameters is a valid course of action (Pfeffer et al. 2008).

Therefore metrics, derived from statistical studies of subjective physical vehicle steering feel assessment, can be applied to quantify the qualitive aspects of steering feel with regards to simulation models.

\subsection{Vehicle steering metrics and characteristics}

Eluded to in previous sections of this paper, physical vehicle steering displays a non-linear, hysteretic profile of torque feedback to the driver. van Daal (2007) cites previous physical studies, such as Farrer (1993) and Chrstos and Grygier (1997) in presenting a basic, graphical definition of 'good' steering feel in terms of time trajectories. These depict the relationship between handwheel angle and steering torque, lateral acceleration and steering torque and finally yaw rate and steering torque. Figures 6, 7 and 8 respectively present the characteristic hysteresis loops.

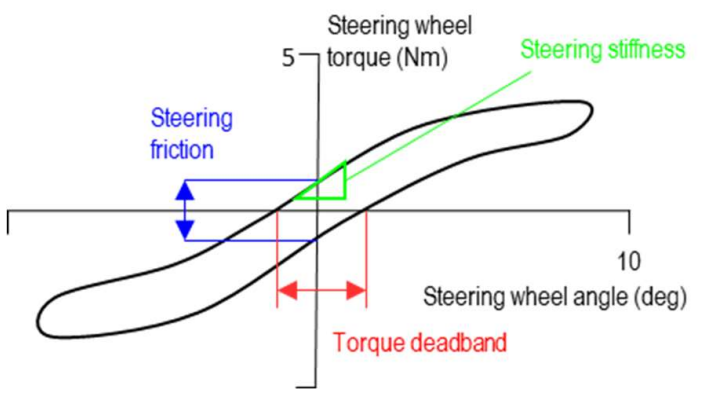

Figure 6. Characteristic hysteresis loop for a steering system. Recreated from van Daal (1997).

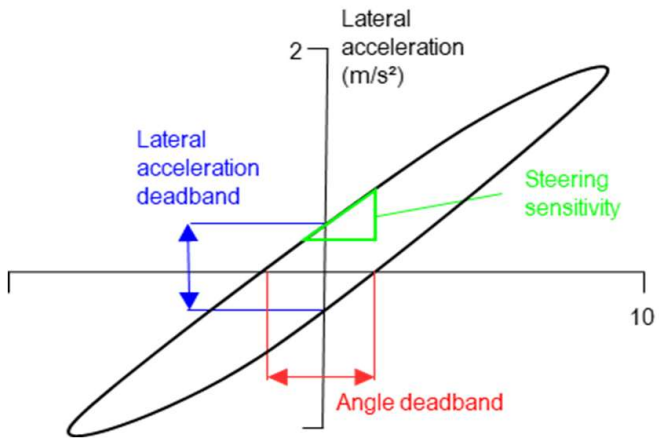

Figure 7. Characteristic hysteresis loop relating the vehicle lateral acceleration response to steering handwheel input Recreated from van Daal (2007).
DOI 


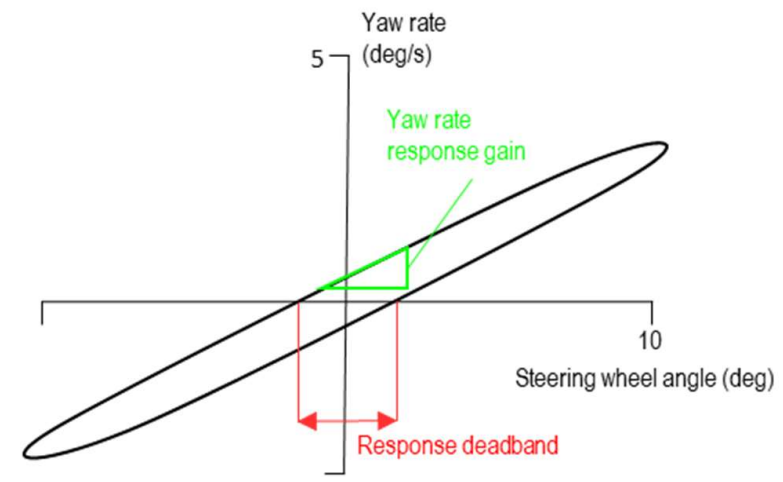

Figure 8. Characteristic hysteresis loop depicting the relationship between vehicle yaw rate response and handwheel input. Recreated from van Daal (2007).

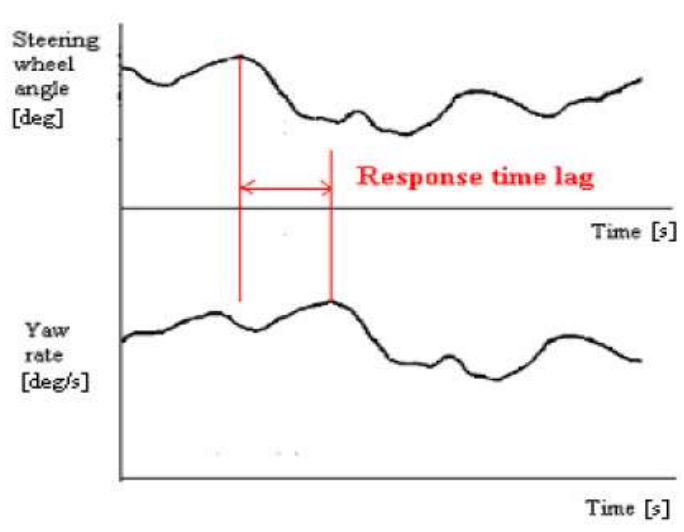

Figure 9. Response lag between steering input and vehicle response, in this case yaw rate (van Daal, 2007).

Of note when considering Figures 6,7 and 8 is that only Figure 6 solely depicts the performance of the steering model in isolation. As Figures 7 and 8 reference vehicle variables (lateral acceleration and yaw rate), it can be deduced that the vehicle platform plays a significant role in producing good steering feel. Various metrics are defined by van Daal (2007) from these plots, which are displayed on Figures 6,7 and 8. Figure 9 presents the lag in time between the steering input and vehicle response. A characteristic such as this is entirely predictable, given basic vehicle dynamics understand pertaining to the transient period of vehicle response.

As van Daal's (2007) work is not specifically focused upon the quantification of what constitutes 'good' steering feel, rather the identification of friction and compliance within the steering system, the application of that study to this paper is limited to holistic consideration of the dynamic relationship between steering angle/torque and vehicle response.

Xuxin and Zhicheng (2012) have built upon van Daal (2007), defining further metrics to characterize steering feel. Specific emphasis in their work is given to the attempt to define and quantify what 'good' steering feels like. A statistical study was conducted on various drivers, with more than 20 vehicles tested across 4 vehicle classes to quantify what drivers considered to be 'good' steering feeling independent of vehicle platform differences.

One upshot of this is Xuxin and Zhicheng (2012) differ in their definitions of basic metrics presented by van Daal (2007), namely the definition of torque deadband. Considering Figure 10 with reference to Figure 6, Xuxin and Zhicheng (2012) calculate the torque deadband angle at $1.3 \mathrm{Nm}$ of steering torque, to define the feeling of play within the steering as experience by a driver. van Daal's (2007) definition of torque deadband is termed as on-center hysteresis by Xuxin and Zhicheng (2012).

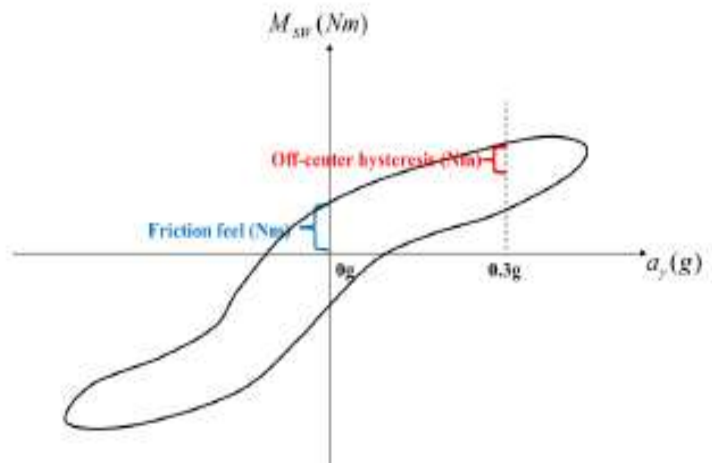

Figure 10. Steering hysteresis loop depicting steering torque against steering angle (Xuxin and Zhicheng, 2012)

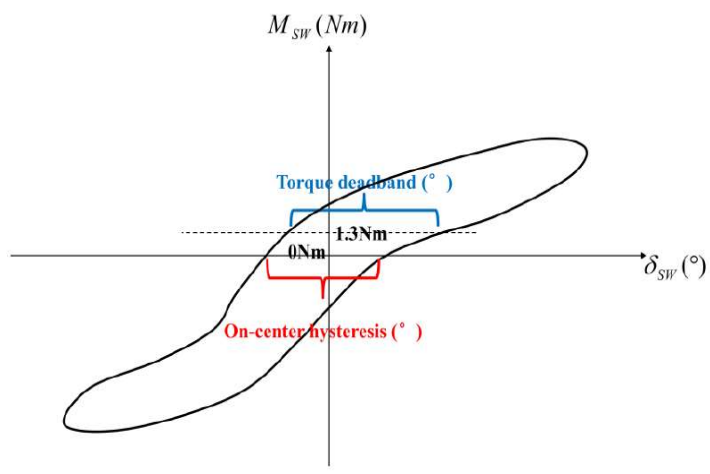

Figure 11. Relationship between steering torque and lateral acceleration, showing the off-center hysteresis (Xuxin and Zhicheng, 2012).

Interestingly, Xuxin and Zhicheng (2012) focus beyond the steering feel during on-center driving, also considering the off-center performance, although oncenter driving appears to form the basis of their study. This is consistent with the Pfeffer et al. (2008) assertion that the majority of road driving concerns on-center steering events. Therefore, Xuxin and Zhicheng (2012) define another metric, off-center hysteresis to define the effort required to correct the steering whilst cornering. This is presented graphically as Figure 11. Whilst a 
formal definition of off-center hysteresis is not given, and the graphical presentation is somewhat unclear, offcenter hysteresis has been interpreted in this paper as meaning the difference between the highest and lowest point of the hysteresis loop at $0.3 \mathrm{~g}$. The written definition of the metric Xuxin and Zhicheng (2012) gives reference to the need to correct the steering, which would entail the steering feedback torque following the lower, return boundary of the hysteresis loop.

Of primary importance to this paper are the quantified values Xuxin and Zhicheng (2012) present regarding what constitutes steering feel with a highly positive rating. Table 1 presents these values.

Table 1. Metric values considered to deliver a 'good' steering feel (Xuxin and Zhicheng, 2012).

\begin{tabular}{|l|l|}
\hline Metric & Range \\
\hline $\begin{array}{l}\text { Response Gain Straight Path } \\
\left(\% / \mathrm{s} / 100^{\circ} \mathrm{SWA}\right)\end{array}$ & $25-30$ \\
\hline Response Time Delay $(\mathrm{m} / \mathrm{s})$ & $>95$ \\
\hline Torque Deadband $\left({ }^{\circ}\right)$ & $<2.2$ \\
\hline Yaw Response Gain $\left(\% / \mathrm{s} / 100^{\circ} \mathrm{SWA}\right)$ & $28-32$ \\
\hline Torque Buildup Cornering $(\mathrm{Nm} / \mathrm{g})$ & $4-6$ \\
\hline Off Center Hysteresis $(\mathrm{Nm})^{\text {Effort Level }(\mathrm{Nm})}$ & $1.5-2.2$ \\
\hline Parking Efforts Standstill $(\mathrm{Nm})$ & $3.6-4.5$ \\
\hline Parking Efforts Rolling $(\mathrm{Nm})$ & $<3.3$ \\
\hline
\end{tabular}

The Response Gain Straight Path is the yaw gain when the vehicle deviates from a straight path (step steer scenario); Yaw Response Gain is the yaw gain during a sinusoidal steering event. Effort level was calculated at $0.3 \mathrm{~g}$ lateral acceleration. Note that these metrics concern both vehicle response variables (relating to the vehicle platform a whole) and metrics directly describing the steering in isolation.

\subsection{Steering model evaluation method}

Knowledge obtained from the research presented in section 3.1 was synthesized to produce a series of Dymola/Modelica experiments. These are designed to evaluate the performance of the steering model presented in this paper against the values in Table 1, and the hysteresis loops in Figures 6, 7 and 8. These experiments enabled the objective assessment of the steering feel in the Dymola simulation environment prior to the model being tested in real-time simulators. Each experiment is described in Table 2. Note, vehicle velocity used was tuned so that other metric conditions (such as specific lateral acceleration, i.e. off-center hysteresis) were met.

All experiments used position actuation of the steering wheel, with the torque feedback (to the simulator rig) visually assessed to be smooth and consistent. Torque values used in metric assessment were handwheel torques, to assess the steering from a driver's viewpoint. Frequency of steering input during testing was $0.2 \mathrm{~Hz}$. A high, medium and low amplitude sinusoidal test was used to evaluate the steering results both with and without engaging the power assistance on-center $\left(< \pm 10^{\circ}\right)$ as well as off-center performance. Closed loop throttle control was used to hold the target vehicle velocity for the tests.

Table 2. Full vehicle experimental setup to evaluate steering model.

\begin{tabular}{|l|l|l|}
\hline Experiment & $\begin{array}{l}\text { Handwheel } \\
\text { angle }\end{array}$ & $\begin{array}{l}\text { Vehicle } \\
\text { velocity }\end{array}$ \\
\hline $\begin{array}{l}\text { Sinusoidal Steer (high } \\
\text { amp) }\end{array}$ & $\pm 30^{\circ}$ & $60 \mathrm{kph}$ \\
\hline $\begin{array}{l}\text { Sinusoidal Steer (mid } \\
\text { amp) }\end{array}$ & $\pm 10^{\circ}$ & $60 \mathrm{kph}$ \\
\hline Sinusoidal Steer (low amp) & $\pm 3^{\circ}$ & $60 \mathrm{kph}$ \\
\hline Stationary Steer & $+150^{\circ}$ & $0 \mathrm{kph}$ \\
\hline Rolling Steer & $+150^{\circ}$ & $7 \mathrm{kph}$ \\
\hline Steering Ramp & $+35^{\circ}$ & $75 \mathrm{kph}$ \\
\hline Step Steer & $+45^{\circ}$ & $45 \mathrm{kph}$ \\
\hline
\end{tabular}

Different metrics were therefore evaluated by different experiments. Table 3 details which metric was evaluated by which test, noting if the metric was required to be evaluated at a specific condition (e.g. specific lateral acceleration).

Table 3. Tests used to evaluate objective metrics.

\begin{tabular}{|l|l|}
\hline Metric & Experiment \\
\hline Response Gain Straight Path & Step Steer \\
\hline Response Time Delay & Sin. Steer (high amp) \\
\hline Torque Deadband & Sin. Steer (low/mid) \\
\hline Yaw Response Gain & Sin. Steer (low/mid) \\
\hline Torque Buildup Cornering & Steering Ramp \\
\hline Off Center Hysteresis & Sin. Steer (high amp) \\
\hline Effort Level & Steering Ramp \\
\hline Parking Efforts Standstill & Stationary Steer \\
\hline Parking Efforts Rolling & Rolling Steer \\
\hline
\end{tabular}

\section{Results}

Table 4 presents assessment of the steering model against the optimal metric values presented in the previous section.

Table 4. Objective metric results from steering model full vehicle testing.

\begin{tabular}{|l|l|}
\hline Metric & Result \\
\hline $\begin{array}{l}\text { Response Gain Straight Path } \\
\left(\% / \mathrm{s} / 100^{\circ} \mathrm{SWA}\right)\end{array}$ & 19.426 \\
\hline Response Time Delay $(\mathrm{m} / \mathrm{s})$ & 80.000 \\
\hline Torque Deadband - mid amplitude $\left({ }^{\circ}\right)$ & 1.661 \\
\hline Torque Deadband - low amplitude $\left({ }^{\circ}\right)$ & 0.125 \\
\hline $\begin{array}{l}\text { Yaw Response Gain - mid amplitude } \\
\left(\% / \mathrm{s} / 100^{\circ} \mathrm{SWA}\right)\end{array}$ & 14.045 \\
\hline $\begin{array}{l}\text { Yaw Response Gain - low amplitude } \\
\left(\% / \mathrm{s} / 100^{\circ} \mathrm{SWA}\right)\end{array}$ & 20.000 \\
\hline
\end{tabular}

DOI 


\begin{tabular}{|l|l|}
\hline Torque Buildup Cornering (Nm/g) & 4.847 \\
\hline Off Center Hysteresis (Nm) & 0.194 \\
\hline Effort Level (Nm) & 4.687 \\
\hline Parking Efforts Standstill (Nm) & 4.694 \\
\hline Parking Efforts Rolling (Nm) & 3.409 \\
\hline
\end{tabular}

Figures 12,13 and 14 detail the characteristic hysteresis loops for the steering model presented in this paper. For direct comparison with Figures 6, 7 and 8, which present the expected appearance, these plots were taken from the Sinusoidal Steer (mid amplitude) simulation. Figures 15 and 16 present the characteristic steering hysteresis loop for the high and low amplitude Sinusoidal Steer tests. To reach these results, the steering model friction parameters (both rotational and translational stiffness, damping and breakaway ratio) were optimized, as well as various power steering parameters such as the emptyingRate, Fdemand, rate and the stiffness/damping of the torsion bar. This was done once the vehicle model has been developed to produce a satisfactory handling performance.

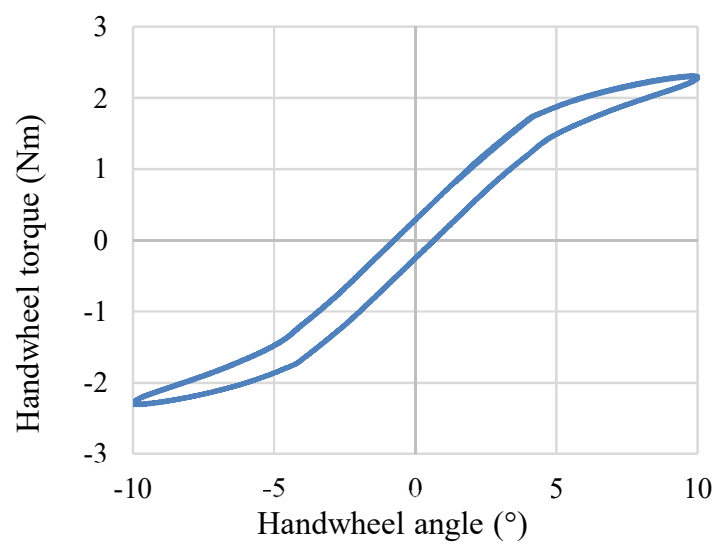

Figure 12. Steering torque hysteresis loop for Sinusoidal Steer test (mid amplitude).

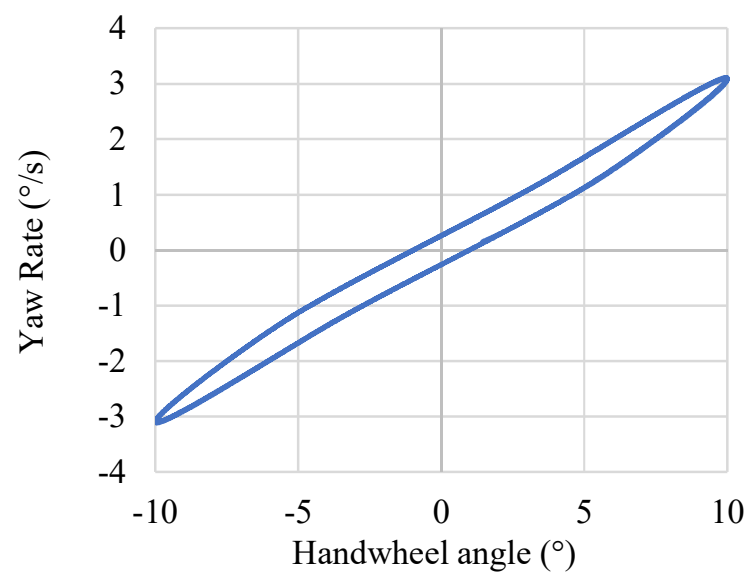

Figure 13. Vehicle yaw response to steering input for the Sinusoidal Steer test (mid amplitude).

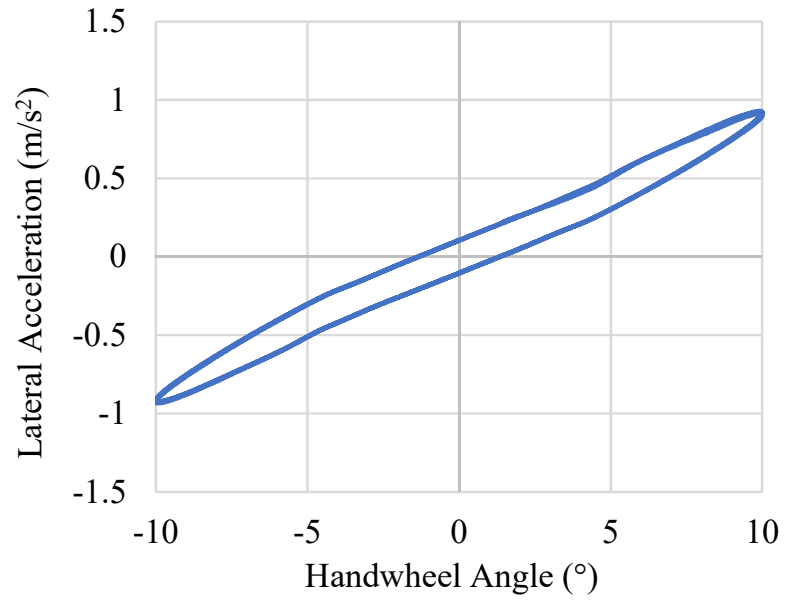

Figure 14. Vehicle lateral acceleration response to steering input for the Sinusoidal Steer test (mid amplitude).

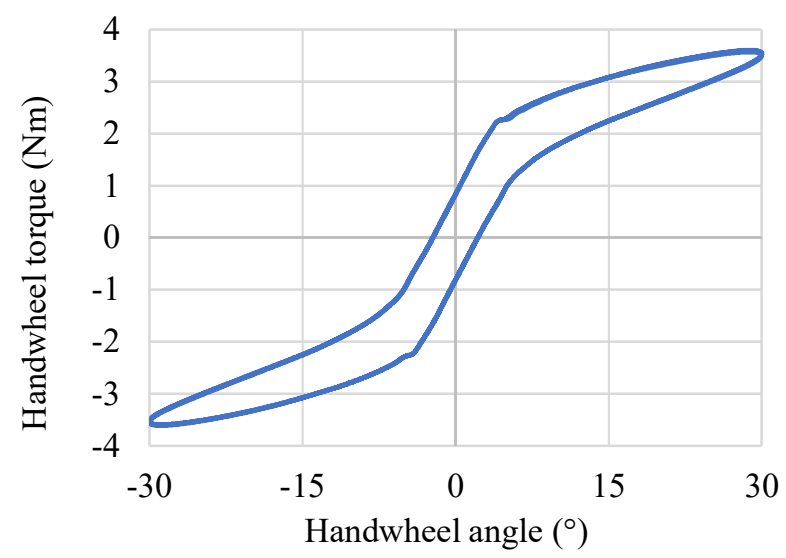

Figure 15. Steering torque hysteresis loop for Sinusoidal Steer test (high amplitude).

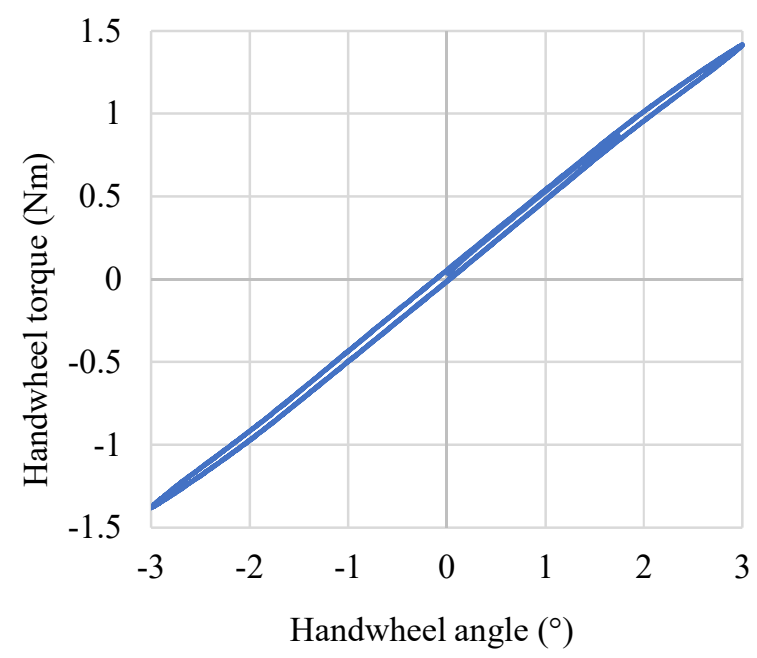

Figure 16. Steering torque hysteresis loop for Sinusoidal Steer test (low amplitude). 


\subsection{Discussion of metrics}

Interrogation of Table 4 with respect to ideal target values detailed in Table 1 indicates the steering model has not achieved the set targets for Response Gain Straight Path, Response Time Delay, Yaw Response Gain and Parking Efforts Standstill. The value calculated for Effort Level it is sufficiently close to the upper boundary to omit discussion into the difference between value and target. Considering the failed metrics, they can be broken up into two distinct groups; metrics indicative of vehicle yaw response (Response Gain Straight Path, Response Time Delay and Yaw Response Gain) and metrics relating to a stationary vehicle condition (Parking Efforts Standstill).

Vehicle yaw response is a function of the vehicle platform as a wider system. Specifically, the performance of the tires and the physical vehicle dynamics setup variables (spring/anti roll bar rates, damper values, mass distribution etc.). Concerning the metrics, Response Time Delay is slightly below the optimal value, meaning the vehicle is achieving peak yaw rate quicker than is ideally desired; the Yaw Response Gains suggest the yaw magnitude for a given steering input is not high enough. Combining these two points, evidence indicates that the vehicle yaw response differs from the vehicles used as part of the Xuxin and Zhicheng (2012) study. Even though that study included cars of this type (executive/E class) as well as larger ones (SUVs), it also included lighter, nimbler vehicles of different classes (small family/C and large family/D class); specific vehicle parameters are not given for the vehicles used in their study. Therefore, it can be expected that the target values their study provides are not totally representative of the class of vehicle used in this study, rather a general idealised target. Thus, it is difficult to draw a direct comparison of vehicle yaw response between the vehicle model used in this study and the vehicles used in Xuxin and Zhicheng (2012). Of further interest, Xuxin and Zhicheng (2012) comment on how they found a lack of correlations in driver feedback for E class/segment vehicles due to a low sample size. Given these points, it is hard to consider the failure to meet the specific vehicle response target metrics to be the result of an incorrect vehicle or steering model, as a direct comparison is problematic. The proximity of the metric scores in this study to the published ideals therefore seems reasonable, indicating the steering model is valid.

Considering the Parking Efforts Standstill result, the source of the failure of achieving this metric can be attributed to the Pacejka (2012) tire model. The Pacejka (2012) model specifically employs an artificial damping factor at zero forward speed to prevenient an undamped vibration from occurring (Pacejka, 2012). It is envisaged that this could be the source of the high comparative magnitude of torque required to move the tire at zero velocity, rather than as a function of the steering model.
Differences in Yaw Response Gain and Torque Deadband results at low/medium amplitudes of steer angle can be considered a result of the experimental setups for each test. The Torque Deadband, being reduced at low steer angles (which do not result in power assistance being applied) suggests most of the hysteresis within the steering is being generated by the power assistance; a claim backed up by the assertion that the rack friction affects the hysteresis less at higher steering wheel angles (Pfeffer et al. 2008). This suggests the power assistance should be dominant over friction in generating hysteresis, which the results indicate. Furthermore, Yaw Response Gain is seen to deteriorate at the mid amplitude steering angle, suggesting the power assistance is adding to the compliance of the steering system during sinusoidal steering, further validating the performance of the pseudo-hydraulic power assistance model.

\subsection{Discussion of plots}

Analysis of Figures 12, 13 and 14 can be considered validation of the generalized performance of the steering model/pseudo-hydraulic power assistance model; the shape and form compare favorably with expected loops of real-world power steering systems presented in Figures 6, 7 and 8. Smooth variation of steering torque in Figure 11 indicates that the friction models are working correctly, with the pronounced bend at $3^{\circ}-5^{\circ}$ steer angle indicating the power assistance is correctly assisting the steering wheel. As the test used to produce Figure 12 utilized a positional input, the feedback torque from the steering model to the handwheel is consistent and smooth, a requirement of DiL steering models. Both Figures 13 and 14 show the vehicle responds correctly to steering input, further reinforcing the view that the vehicle model is valid, with the difference in the yaw metric scores is due to the yaw response metrics being not directly applicable to a vehicle of this class.

Moving onto Figure 15, a Sinusoidal Steer test of up to $30^{\circ}$, the same general hysteresis loop shape is presented as in Figure 12, a test of up to $10^{\circ}$ steer. In effect this plot confirms the general validity of the steering model presented, as the same characteristics are present during both on and off-center steering situations. Noting the slight notch as the steering moves from unassisted to power assisted; not present in Figure 12, it can be deduced that this is an artefact of friction model/power assistance interaction at that point. This is most likely to be the result of non-optimal parameterization rather than model deficiencies, due to the small localized nature and the absence of a similar notch on the return bound of the hysteresis loop. Figure 16 , where the power assistance is not engaged, supports this hypothesis, as the friction model torque response is smooth and consistent, with both the stationary and dynamic states encountered by the models. Of final note is the comparative lack of hysteresis shown in Figure 16,
DOI 
reflected in the Torque Deadband result for the Sinusoidal Steer test (low amplitude). This indicates that the current parameterization of the steering model does not include enough friction force, explaining the lack of hysteresis shown at each end of the steering torque hysteresis loops in Figures 12 and 15, and indicating this phenomenon is the result of non-optimal friction model parameterization rather than a modeling error.

\subsection{Comments on real-time running}

Various parameterization setups of the steering model (friction settings, steering assistance levels) were tested successfully on a workstation DiL setup before being deployed successfully on a full DiL simulator rig.

\section{Conclusions}

Overall, the work conducted during this study supports the presentation of the pseudo-hydraulic steering model as applicable for DiL applications for both on-center and off-center driving, based primarily on the generation of feedback curves characteristic of a real-world physical steering system. Steering model parameters were adjusted to influence the steering feel. The model has been shown to produce a representative vehicle and torque feedback response, comparing favorably with idealized objective metrics derived from physical vehicle testing. This indicates that the pseudo-hydraulic power assistance block is functioning correctly, with the friction model selection being valid. Whilst evidence indicates that the parameterization of the steering model is not optimal, this paper serves as a proof of concept of the pseudo-hydraulic steering model presented.

\subsection{Further work}

Initial further work would pertain to the analysis of free steer capability/performance of the steering model, as one of key advantage of using a pseudo-hydraulic power assistance model is the decay rate of force when the torque applied drops off suddenly. This would be using the methods/targets in ISO 17288:2011. Following this, obtaining a dataset of a real physical vehicle (dynamic setup etc.), would enable a parameterization setup to be developed with further confidence.

Developing a physical model of a hydraulic power steering would be of interest. The VeSyMA approach to the vehicle and subsystem architecture enables comparison between different implementations of the same system. Such a comparison between a physical model and the pseudo-hydraulic model could be used to fit the parameters for the pseudo-hydraulic model to suit a specific design, so that the design can be evaluated in a DiL environment.

\section{References}

Johan Andreasson, Naoya Machida, Masashi Tsushima, John Griffin and Peter Sundström (2016). Deployment of highfidelity vehicle models for accurate real-time simulation.
Proceedings of $1^{\text {st }}$ Japanese Modelica Conference, pp7886, 2016. DOI:10.3384/ecp1612495

Ansible Motion: Looking Down the Road: Driving Simulator Technology and How Automotive Manufacturers Will Benefit. White paper, 2015.

J.P. Chrstos, P.A. Grygier (1997). Experimental Testing of a 1994 Ford Taurus for NADSdyna Validation. SAETechnical Paper 970563, 1997.

H. Dankowicz (1999). Modelling of dynamic friction phenomena. ZAMM 1999;79:399-409.

Pierre Dupont, Vincent Hayward, Brian Armstrong and Friedhelm Altpeter (2002). Single state elastoplastic friction models. Automatic Control, IEEE Transactions on Automatic Control, 47.5 (2002): 787-792.

D.G. Farrer (1993). An Objective Measurement Technique for the Quantification of On-Centre Handling Quality, $S A E$ Technical Paper 930827, 1993.

Hans B. Pacejka (2012). Tyre and Vehicle Dynamics, $3^{\text {nd }}$ edition. Oxford: Elsevier, pp 356-404.

Nissan. 2018. Electro-Hydraulic power steering system. Online. Accessed 18 March 2018. Available at: https://www.nissanglobal.com/EN/TECHNOLOGY/OVERVIEW/ehpss.html

P. E. Pfeffer, M. Harrer and D.N Johnston (2008). Interaction of vehicle and steering regarding on-centre handling. Vehicle System Dynamics: International Journal of Vehicle Mechanics and Mobility, 46:5, 413-428, DOI: 10.1080/00423110701416519

Marcus Rösth (2009). Hydraulic Power Steering System Design in Road Vehicles. Doctoral Thesis, Department of Management and Engineering, Linköping University, 2009.

B.A.M van Daal (2007). Friction and compliance identification in a vehicle's steering system. Extern traineeship report, Eindhoven University of Technology, 2007.

Xuxin He and Zhicheng Su (2012). Links between Subjective Assessments and Objective Metrics for Steering. Master's Thesis, KTH Royal Institute of Technology, Stockholm Sweden, 2012. 